\title{
Comunicaciones recibidas con posterioridad a la impresión del volumen 10-3 de Educación Médica.
}

\author{
B-13 COMPETENCIAS, ACTIVIDADES \\ DE APRENDIZAJE Y EVALUACIÓN; \\ UNA COHERENCIA IMPRESCINDIBLE \\ EN EL DISEÑO DE LA ASIGNATURA \\ ENFERMERÍA MÉDICO-QUIRÚRGICA \\ ADULTO-I. \\ Fernández P., Edo M. y Fernández L. Escuela \\ Universitaria Enfermería Gimbernat, adscrita UAB
}

La construcción del Espacio Europeo de Educación Superior implica diseños curriculares en los que se establezca una coherencia entre las competencias demandadas, las actividades de aprendizaje propuestas y los sistemas de evaluación que demuestran su consecución. En este sentido, el trabajo que presentamos pretende explicar la adaptación de la asignatura troncal Enfermería Médico-Quirúrgica Adulto-I dentro del currículo de la titulación de Enfermería. La implementación se ha realizado durante el curso 2006-2007, en el marco del Plan Piloto de Adaptación de Titulaciones del Departament d'Educació i Universitats en el que participa la E.U.E. Gimbernat. El diseño de la asignatura se ha realizado en base a las etapas:1) Consenso del equipo de profesores de la asignatura respecto a la elección de las competencias pertinentes para trabajar en la asignatura 2) Reflexión en torno a la elaboración de las actividades de aprendizaje y su adecuación a las competencias 3) Temporalización de las actividades dentro del plan de aprendizaje y distribución de la carga de trabajo del estudiante y 4) Elaboración de una propuesta de evaluación coherente.

\section{C-11 ABP INTEGRAL EN HISTOLOGÍA (TEORÍA Y PRÁCTICAS), UNA EXPERIENCIA EXITOSA EN LA UAB. \\ Martín V., Castellano B., González B. y Acarín L. Facultad de Medicina, Universidad Autónoma de Barcelona}

En nuestra unidad y durante varios cursos académicos hemos desarrollado una metodología docente basada en ABP.
Al principio del curso se propone un exhaustivo programa de objetivos de conocimientos y habilidades, sobre este programa se desarrolla la asignatura.

En teoría, cada sesión de aula se desarrolla como sesión de discusión de puntos del programa definidos y que los alumnos deben preparar de forma previa a la sesión, para ayudar a su preparación los profesores responsables "cuelgan" del Campus Virtual materiales de referencia, interactivos, etc...

Durante la sesión y para conseguir una participación del mayor número de estudiantes, se reparten (en grupos de 3-4) láminas que contienen uno o varios casos, que incluyen imágenes, esquemas, preguntas, etc. Los estudiantes disponen de 15-20 minutos para resolverlos, posteriormente son discutidos en conjunto. Los casos se han diseñado para comprobar la comprensión de los conceptos incluidos en el tema y sirven para que el docente conduzca la discusión.

La última incorporación al método es el uso de un laboratorio digital de microscopía para realizar un sistema similar en las sesiones prácticas.

\section{C-12 LA ENSEÑANZA TEÓRICA DE LA MEDICINA DESDE LA PERSPECTIVA DEL ALUMNO. ESTUDIO EN LA UNVERSIDAD DE SALAMANCA \\ Méndez C., Sánchez-Ledesma MJ., Pérez de la Cruz, MA. y López-Sosa C. \\ Facultad de Medicina. Universidad de Salamanca}

Objetivos: Aproximarnos al valor que tiene actualmente la docencia teórica para los alumnos, así como la importancia que el propio estudiante le concede a un mayor desarrollo del aprendizaje dirigido.

Metodología: Hemos realizado un estudio descriptivo mediante cuestionario anónimo a alumnos de la titulación de Medicina de Salamanca. La muestra está formada por 488 estudiantes desde primero a sexto curso. Las cuestiones planteadas hacen referencia a aspectos relativos a la docencia teórica que reciben en el aula así como a aquellos otros implicados en la organización de la misma. Se 
analizaron variables como el tiempo empleado en preparar clases, utilización como apoyo de material complementario y los servicios bibliográficos e intervención de los alumnos en la preparación y desarrollo de las clases teóricas.

Resultados: Más del 60\% afirman que las clases magistrales proporcionan información resumida y actualizada sobre un tema y las consideran necesarias para un buen aprendizaje (44\%). Valoran que los medios audiovisuales son adecuados, facilitan el estudio y se ajustan a los objetivos planteados. Más del $63 \%$ en sexto curso afirman que los profesores no dedican parte del tiempo de la clase a responder a sus preguntas y ellos ante una duda surgida en clase no preguntan habitualmente a su profesor. Los alumnos no dedican un tiempo determinado para la preparación previa de las clases, más del $50 \%$ emplean menos de 6 horas semanales en completar sus clases teóricas mediante la consulta de libros y de la red.

Conclusiones: La hegemonía de la docencia teórica y más concretamente de la clase magistral en la docencia en Medicina cuenta con la aprobación evidente de los estudiantes.

\section{E- 9 LABORATORIO VIRTUAL DE MICROSCOPIA, UNA HERRAMIENTA DOCENTE EFICAZ.}

Martín V., Castellano B., González B. y Acarín L. Facultad de Medicina, Universidad Autónoma de Barcelona

La docencia de la Histología, así como otras materias afines, basa su parte práctica en el estudio al microscopio de numerosas preparaciones.

En nuestra unidad, y después de muchos esfuerzos, hemos conseguido que los estudiantes puedan disponer de un moderno laboratorio de microscopía, dotado de numerosos microscopios (42) y una surtida histoteca, pero aún así puede resultar insuficiente, dado el gran número de estudiantes matriculados en las asignaturas que impartimos (387 en Histología Médica de $1^{\circ}$ y 298 en Estructura Microscópica de Aparatos y Sistemas Humanos de $2^{\circ}$ en el curso 2006-07).

Para solventar esta situación hemos participado en el desarrollo de un simulador informático que permite al estudiante realizar las mismas tareas que un laboratorio convencional de microscopia pero en un ordenador: el "Laboratorio Virtual de Microscopía"; a efectos prácticos es como si el estudiante dispusiera en su casa de un microscopio y una completa histoteca. El trabajo con el simulador se completa con sesiones de refuerzo y discusión en el laboratorio.
Hemos usado este sistema en el presente curso con unos resultados francamente positivos.

\section{F-9 ESTUDIO COMPARATIVO ENTRE DISTINTOS MÉTODOS DE EVALUACIÓN DE CONOCIMIENTOS}

Leaman O. ${ }^{-}$, Álvarez de Sierra B. ${ }^{1}$, Sánchez A. ${ }^{2}$, Rodríguez LF. , López-Herce JA ${ }^{1}$, Vigil MD., ${ }^{2} y$ Millán J. ${ }^{1}$ HGU Gregorio Marañón. ${ }^{1}$ Cátedra Fundación Lilly-UCM de Educación Médica. ${ }^{2}$ $S^{o}$ Med. Prev. y Gestión de Calidad.

Existen diversos métodos para valorar los conocimientos de un estudiante.

Objetivo: Comparar tres métodos de evaluación: Test de respuesta única cierta, Preguntas cortas y desarrollo de un Tema.

Material y métodos: Estudio transversal con componentes analíticos. Población: 100 alumnos de una promoción de Medicina. Variables: puntuaciones obtenidas en ocho materias del programa de Patología Médica, impartidas a lo largo de tres años consecutivos. La extensión, el tiempo y el corrector fueron los mismos. Se realizó un análisis de la concordancia entre los tres métodos de evaluación mediante el coeficiente de correlación intraclase (CCI) completándose con el análisis de diferencias individuales: método Bland-Altman.

Resultados: 1) Los tres métodos de evaluación son moderadamente concordantes en la mayoría de las materias (CCI:0.51- 0.70). 2) El grado de concordancia varía en función de la materia. Nefrología presenta el menor CCI mientras que en Hematología y Neumología los valores son mayores. 3) Las diferencias de las puntuaciones obtenidas con el test menos el tema son positivas con menor nota media y a la inversa.

\section{G-9 EVALUACIÓN DE LA IMPLEMENTACIÓN DE LA ASIGNATURA ENFERMERÍA MÉDICO-QUIRÚRGICA ADULTO-I: DOS PERSPECTIVAS CONVERGENTES}

Edo M., Fernández L. y Fernández P. Escuela Universitaria Enfermería Gimbernat, adscrita UAB

Se presentan los resultados de la evaluación de la implementación de la asignatura Enfermería MédicoQuirúrgica Adulto-I, implementada durante el curso 2006-07 siguiendo las directrices del Plan Piloto para la Adaptación de las Titulaciones al Espacio Europeo de Educación Superior del Departament d'Educació i Universitats de la Generalitat de Catalunya.

La evaluación se basa en la valoración de la percepción respecto al proceso de implementación 
desde dos perspectivas diferenciadas; estudiantes y profesores de la asignatura. Los datos se han obtenido a través de cuestionarios diseñados específicamente; los estudiantes valoraban aspectos relacionados con las actividades de aprendizaje, los mecanismos de evaluación, la actuación docente del profesorado, el desarrollo de las competencias y el grado de satisfacción global. Por otra parte, el profe- sorado expresaba su percepción en torno al diseño, desarrollo y resultados de la implementación de la asignatura.

En cuanto a resultados, cabe destacar el alto grado de satisfacción global, la notable percepción por parte de los estudiantes respecto a la adquisición de las competencias y la evidencia del aumento de la carga de trabajo para el profesorado. 